\title{
Conservation Laws, Symmetry Reductions, and New Exact Solutions of the $(2+1)$-Dimensional Kadomtsev-Petviashvili Equation with Time-Dependent Coefficients
}

\author{
Li-hua Zhang \\ Department of Mathematics, Dezhou University, Dezhou 253023, China \\ Correspondence should be addressed to Li-hua Zhang; fim100@163.com
}

Received 16 November 2013; Revised 25 January 2014; Accepted 19 February 2014; Published 9 April 2014

Academic Editor: Mariano Torrisi

Copyright (C) 2014 Li-hua Zhang. This is an open access article distributed under the Creative Commons Attribution License, which permits unrestricted use, distribution, and reproduction in any medium, provided the original work is properly cited.

The $(2+1)$-dimensional Kadomtsev-Petviashvili equation with time-dependent coefficients is investigated. By means of the Lie group method, we first obtain several geometric symmetries for the equation in terms of coefficient functions and arbitrary functions of $t$. Based on the obtained symmetries, many nontrivial and time-dependent conservation laws for the equation are obtained with the help of Ibragimov's new conservation theorem. Applying the characteristic equations of the obtained symmetries, the $(2+1)$-dimensional KP equation is reduced to $(1+1)$-dimensional nonlinear partial differential equations, including a special case of $(2+1)$-dimensional Boussinesq equation and different types of the KdV equation. At the same time, many new exact solutions are derived such as soliton and soliton-like solutions and algebraically explicit analytical solutions.

\section{Introduction}

The Lie group method is a powerful tool to perform Lie symmetry analysis, study conservation laws, and look for exact solutions of nonlinear partial differential equations (NLPDEs) [1-4]. The notion of conservation laws, which plays an important role in the study of nonlinear science, is used for the development of appropriate numerical methods and for mathematical analysis, in particular, existence, uniqueness, and stability analysis $[5,6]$. In addition, the existence of a large number of conservation laws of a partial differential equation (system) is a strong indication of its integrability. On the other hand, seeking exact solutions of NLPDEs has become one central theme of perpetual interest in mathematical physics as explicit solutions will be helpful to better understand the phenomena described by the equations. To get exact solutions of NLPDEs, many effective methods have been presented such as inverse scattering method [7], Hirota's bilinear method [8], and Painlevé expansion method [9]. Among them the Lie group method offers a systematic algorithmic procedure to find the symmetry reductions and exact solutions of a partial differential equation. In this paper, we use the Lie group method to consider a time-dependent Kadomtsev-Petviashvili equation:

$$
E_{1} \equiv u_{x t}+6 u_{x}^{2}+6 u u_{x x}+u_{x x x x}+e(t) u_{x}+n(t) u_{y y}=0,
$$

with time-dependent coefficient functions $e(t), n(t)$, and $n(t) \neq 0$.

The above equation was also called "a $2 \mathrm{D} \mathrm{KdV}$ equation with time-dependent coefficients" by Hereman and Zhuang [10]; they performed Painlevé analysis for (1) and found that (1) was Painlevé integrable when $e_{t}+2 e^{2}=0, n_{t}+4 n e=$ 0 . Equation (1) can be reduced to the $\mathrm{KdV}$ equation $(e(t)=$ $0, n(t)=0)$ or the KP equation $(e(t)=0, n(t)= \pm 1)$. Equation (1) can also be reduced to the cylindrical KdV equation

$$
u_{t}+6 u u_{x}+u_{x x x}+\frac{1}{2 t} u=0,
$$

when $e(t)=1 / 2 t, n(t)=0$ or the cylindrical KP equation

$$
u_{x t}+6 u_{x}^{2}+6 u u_{x x}+u_{x x x x}+\frac{1}{2 t} u_{x} \pm 3 \frac{1}{t^{2}} u_{y y}=0,
$$


when $e(t)=1 / 2 t, n(t)= \pm 3 / t^{2}$. The KdV and KP equations and their cylindrical generalizations $(2 \mathrm{a})$ and $(2 \mathrm{~b})$ are all known to be completely integrable [10]. Zhang et al. [11] performed Painlevé analysis for (1) and constructed bilinear auto-Bäcklund, analytic solutions in the Wronskian form. Soliton-like solutions, Jacobi elliptic function-like solutions, and other exact solutions have been obtained by the method of auxiliary equations [12-15]. Elwakil et al. [16] used the homogeneous balance method to study the exact solutions of (1). Based on the homogeneous balance method and Clarkson-Kruskal method, direct reduction and exact solutions have been obtained in [17] by Moussa and El-Shiekh. The bilinear formalism, bilinear Bäcklund transformation, and Lax pair of (1) have been obtained by the binary Bell polynomial approach in [18]. As far as we know, conservation laws and symmetry reductions for (1) have not been studied.

The rest of the paper is organized as follows. In Section 2, the Lie group method is applied to the time-dependent Kadomtsev-Petviashvili equation (1) and thus Lie symmetries of (1) are obtained. In Section 3, using the obtained symmetries and the general theorem on conservation laws by Ibragimov, nontrivial and time-dependent conservation laws are derived. In Section 4, we use the symmetry to get symmetry reductions and new exact solutions of (1). The last section is a short summary and discussion.

\section{Lie Symmetry Analysis of (1)}

Generally speaking, Lie symmetry denotes a transformation that leaves the solution manifold of a system invariant; that is, it maps any solution of the system into a solution of the same system, so it is also called geometric symmetry. In this section, we will perform Lie symmetry analysis for (1) by the classical Lie group method. Suppose that Lie symmetry of (1) is expressed as follows:

$$
V=\xi \frac{\partial}{\partial x}+\eta \frac{\partial}{\partial y}+\tau \frac{\partial}{\partial t}+\phi \frac{\partial}{\partial u}
$$

where $\xi, \eta, \tau$, and $\phi$ are undetermined functions with respect to $x, y, t$, and $u$. According to the procedures of Lie group method, the vector field (3) can be determined by applying the fourth prolongation of $V$ to (1) and thus the undetermined functions $\xi, \eta, \tau$, and $\phi$ must satisfy the following invariant condition:

$$
\begin{aligned}
\phi^{x t} & +12 u_{x} \phi^{x}+6 u_{x x} \phi+6 u \phi^{x x}+\phi^{x x x x} \\
& +e^{\prime}(t) \tau u_{x}+e(t) \phi^{x}+n^{\prime}(t) \tau u_{y y}+n(t) \phi^{y y}=0,
\end{aligned}
$$

where

$$
\begin{gathered}
\phi^{x}=D_{x}\left(\phi-\xi u_{x}-\eta u_{y}-\tau u_{t}\right)+\xi u_{x x}+\eta u_{x y}+\tau u_{x t}, \\
\phi^{x t}=D_{x t}\left(\phi-\xi u_{x}-\eta u_{y}-\tau u_{t}\right)+\xi u_{x x t}+\eta u_{x t y}+\tau u_{x t t},
\end{gathered}
$$

$$
\begin{gathered}
\phi^{x x}=D_{x x}\left(\phi-\xi u_{x}-\eta u_{y}-\tau u_{t}\right) \\
+\xi u_{x x x}+\eta u_{x x y}+\tau u_{x x t}, \\
\phi^{y y}=D_{y y}\left(\phi-\xi u_{x}-\eta u_{y}-\tau u_{t}\right) \\
+\xi u_{x y y}+\eta u_{y y y}+\tau u_{y y t}, \\
\phi^{x x x x}=D_{x x x x}\left(\phi-\xi u_{x}-\eta u_{y}-\tau u_{t}\right) \\
+\xi u_{x x x x x}+\eta u_{x x x x y}+\tau u_{x x x x t} .
\end{gathered}
$$

Substituting (5) into (4) with $u$ being a solution of (1), that is,

$$
u_{x x x x}=-u_{x t}-6 u_{x}^{2}-6 u u_{x x}-e(t) u_{x}-n(t) u_{y y},
$$

we obtain the determining equations of symmetry (3). Solving the determining equations with the aid of Maple, we can get the following cases.

Case 1. When $e(t)$ and $n(t)$ are arbitrary functions,

$$
\begin{gathered}
\xi=-\frac{g_{t} y}{2 n(t)}+f(t), \quad \eta=g(t), \quad \tau=0, \\
\phi=\frac{f_{t}}{6}-\frac{g_{t t}}{12 n(t)} y+\frac{g_{t} n_{t}}{12 n^{2}(t)} y,
\end{gathered}
$$

where $f(t)$ and $g(t)$ are arbitrary functions. It shows that (1) admits an infinite-dimensional Lie algebra of symmetries

$$
V=V_{f}+V_{g}
$$

where

$$
\begin{gathered}
V_{f}=f(t) \frac{\partial}{\partial x}+\frac{f_{t}}{6} \frac{\partial}{\partial u}, \\
V_{g}=-\frac{g_{t} y}{2 n(t)} \frac{\partial}{\partial x}+g(t) \frac{\partial}{\partial y}+\left(\frac{g_{t} n_{t}}{12 n^{2}(t)} y-\frac{g_{t t}}{12 n(t)} y\right) \frac{\partial}{\partial u} .
\end{gathered}
$$

Case 2. When $e(t)=0, n(t)=(t-m)^{p} C_{1}, p \neq 0, C_{1} \neq 0$, and $\mathrm{C}_{2} \neq 0$,

$$
\begin{gathered}
\xi=\frac{C_{2} x}{3 p}-\frac{g_{t} y}{2 C_{1}(t-m)^{p}}+f(t), \\
\eta=\left(\frac{2 C_{2}}{3 p}+\frac{C_{2}}{2}\right) y+g(t), \quad \tau=\frac{C_{2}(t-m)}{p}, \\
\phi=-\frac{2 C_{2}}{3 p} u+\frac{g_{t}}{12 C_{1}(t-m)^{p+1}} y p-\frac{g_{t t}}{12 C_{1}(t-m)^{p}} y+\frac{f_{t}}{6},
\end{gathered}
$$

where $m, p, C_{1}$, and $C_{2}$ are constants and $f(t)$ and $g(t)$ are arbitrary functions. This shows that the symmetries of equation

$$
u_{x t}+6 u_{x}^{2}+6 u u_{x x}+u_{x x x x}+C_{1}(t-m)^{p} u_{y y}=0
$$


have the form of

$$
V=V_{1}+V_{f}+V_{g}
$$

where

$$
V_{1}=\frac{x}{3 p} \frac{\partial}{\partial x}+\left(\frac{2}{3 p}+\frac{1}{2}\right) y \frac{\partial}{\partial y}+\frac{(t-m)}{p} \frac{\partial}{\partial t}-\frac{2}{3 p} u \frac{\partial}{\partial u}
$$

is a one-dimensional Lie algebra of symmetries and $V_{f}$ and $V_{g}$ are two infinite-dimensional Lie algebra of symmetries as expressed by (9) with $n(t)=(t-m)^{p} C_{1}$.

Case 3. When $e(t)=0, n(t)=$ Const., and $\tau(t) \neq 0$,

$$
\begin{gathered}
\xi=\frac{\tau_{t}}{3} x-\frac{\tau_{t t}}{6 n} y^{2}-\frac{g_{t}}{2 n} y+f(t), \\
\eta=\frac{2}{3} \tau_{t} y+g(t), \quad \tau=\tau(t), \\
\phi=-\frac{2 \tau_{t}}{3} u+\frac{\tau_{t t}}{18} x-\frac{\tau_{t t t}}{36 n} y^{2}-\frac{g_{t t}}{12 n} y+\frac{f_{t}}{6},
\end{gathered}
$$

where $f(t)$ and $g(t)$ are arbitrary functions. It shows that the KP equation

$$
u_{x t}+6 u_{x}^{2}+6 u u_{x x}+u_{x x x x}+C u_{y y}=0
$$

admits an infinite-dimensional Lie algebra of symmetries

$$
V=V_{f}+V_{g}+V_{\tau}
$$

where $C$ is a constant and $C \neq 0 ; V_{f}$ and $V_{g}$ are expressed by (9) with $n(t)=$ Const.,

$$
\begin{aligned}
V_{\tau}= & \left(\frac{\tau_{t}}{3} x-\frac{\tau_{t t}}{6 n} y^{2}\right) \frac{\partial}{\partial x}+\frac{2}{3} \tau_{t} y \frac{\partial}{\partial y}+\tau \frac{\partial}{\partial t} \\
& +\left(-\frac{2 \tau_{t}}{3} u+\frac{\tau_{t t}}{18} x-\frac{\tau_{t t t}}{36 n} y^{2}\right) \frac{\partial}{\partial u} .
\end{aligned}
$$

Case 4. When $e(t)=-n_{t} / 4 n+C_{3} / \tau(t), \tau(t) \neq 0$, and $n_{t} \neq 0$,

$$
\begin{aligned}
\xi= & \frac{\tau_{t}}{3} x-\frac{\tau_{t t}}{6 n(t)} y^{2}-\frac{g_{t}}{2 n(t)} y-\frac{\tau_{t} n_{t}}{8 n^{2}(t)} y^{2} \\
& -\frac{\tau(t) n_{t t}}{8 n^{2}(t)} y^{2}+\frac{\tau(t) n_{t}^{2}}{8 n^{3}(t)} y^{2}+f(t),
\end{aligned}
$$

$$
\begin{gathered}
\eta=\left(\frac{\tau(t) n_{t}}{2 n(t)}+\frac{2}{3} \tau_{t}\right) y+g(t), \\
\phi=-\frac{2 \tau_{t}}{3} u+\frac{\tau_{t t}}{18} x+\frac{\tau(t) n_{t t} n_{t}}{12 n^{3}(t)} y^{2} \\
-\frac{\tau(t) n_{t t t}}{48 n^{2}(t)} y^{2}-\frac{\tau(t) n_{t}^{3}}{16 n^{4}(t)} y^{2}+\frac{\tau_{t t} n_{t}}{144 n^{2}(t)} y^{2} \\
-\frac{\tau_{t t t}}{36 n(t)} y^{2}+\frac{\tau_{t} n_{t}^{2}}{16 n^{3}(t)} y^{2}-\frac{\tau_{t} n_{t t}}{24 n^{2}(t)} y^{2} \\
+\frac{f_{t}}{6}-\frac{g_{t t}}{12 n(t)} y+\frac{g_{t} n_{t}}{12 n^{2}(t)} y,
\end{gathered}
$$

where $f(t)$ and $g(t)$ are arbitrary functions, $C_{3}$ is an integral constant, and $n(t)$ and $\tau(t)$ satisfy the following ordinary differential equation:

$$
\begin{gathered}
n_{t t t}+\frac{2 n_{t t} \tau_{t}}{\tau(t)}-\frac{3 \tau_{t} n_{t}^{2}}{n(t) \tau(t)}+\frac{3 n_{t}^{3}}{n^{2}(t)} \\
-\frac{4 n_{t t} n_{t}}{n(t)}-\frac{4 C_{3} n(t) \tau_{t t}}{3 \tau^{2}(t)}=0 .
\end{gathered}
$$

This shows that, under the condition (19), the equation

$$
\begin{aligned}
u_{x t} & +6 u_{x}^{2}+6 u u_{x x}+u_{x x x x} \\
& +\left(-\frac{n_{t}}{4 n}+\frac{C_{3}}{\tau(t)}\right) u_{x}+n(t) u_{y y}=0
\end{aligned}
$$

admits an infinite-dimensional Lie algebra of symmetries

$$
V=V_{f}+V_{g}+V_{0 \tau}
$$

where $V_{f}$ and $V_{g}$ are expressed by (9):

$$
\begin{aligned}
V_{0 \tau}=\left(\frac{\tau_{t}}{3} x-\frac{\tau_{t t}}{6 n} y^{2}-\frac{\tau_{t} n_{t}}{8 n^{2}(t)} y^{2}-\frac{\tau(t) n_{t t}}{8 n^{2}(t)} y^{2}\right. \\
\left.+\frac{\tau(t) n_{t}^{2}}{8 n^{3}(t)} y^{2}\right) \frac{\partial}{\partial x}+\left(\frac{\tau(t) n_{t}}{2 n(t)}+\frac{2}{3} \tau_{t}\right) y \frac{\partial}{\partial y}+\tau \frac{\partial}{\partial t} \\
+\left(-\frac{2 \tau_{t}}{3} u+\frac{\tau_{t t}}{18} x+\frac{\tau(t) n_{t t} n_{t}}{12 n^{3}(t)} y^{2}-\frac{\tau(t) n_{t t t}}{48 n^{2}(t)} y^{2}\right. \\
-\frac{\tau(t) n_{t}^{3}}{16 n^{4}(t)} y^{2}+\frac{\tau_{t t} n_{t}}{144 n^{2}(t)} y^{2}-\frac{\tau_{t t t}}{36 n(t)} y^{2} \\
\left.+\frac{\tau_{t} n_{t}^{2}}{16 n^{3}(t)} y^{2}-\frac{\tau_{t} n_{t t}}{24 n^{2}(t)} y^{2}\right) \frac{\partial}{\partial u} .
\end{aligned}
$$

\section{Conservation Laws for (1)}

3.1. A General Theorem on Conservation Laws. As expressed through the famous Noether theorem, for a given differential equation, there is a close connection between Lie symmetries and conservation laws. To derive conservation laws of (1), we use the following conclusion proved by Ibragimov in [19]. 
Theorem 1. Every Lie point, Lie-Bäcklund, and nonlocal symmetry

$$
V=\xi^{i}\left(x, u, u_{(1)}, \ldots\right) \frac{\partial}{\partial x^{i}}+\eta^{s}\left(x, u, u_{(1)}, \ldots\right) \frac{\partial}{\partial u^{s}}
$$

of a system of $m$ equations

$$
F_{s}\left(x, u, u_{(1)}, \ldots, u_{(N)}\right)=0, \quad s=1, \ldots, m
$$

with $n$ independent variables $x=\left(x^{1}, \ldots, x^{n}\right)$ and $m$ dependent variables; $u=\left(u^{1}, \ldots, u^{m}\right)$ provides a conservation law for system (24) and the corresponding adjoint system

$$
\begin{gathered}
F_{s}^{*}\left(x, u, v, u_{(1)}, v_{(1)}, \ldots, u_{(N)}, v_{(N)}\right) \\
\equiv \frac{\delta\left(v^{i} F_{i}\right)}{\delta u^{s}}=0, \quad s=1, \ldots, m .
\end{gathered}
$$

Then the elements of the conservation vector $T=\left(T^{1}, \ldots, T^{n}\right)$ are defined by the following expression:

$$
\begin{aligned}
T^{i}= & \xi^{i} L+W^{s} \\
& \times\left[\frac{\partial L}{\partial u_{i}^{s}}-D_{x^{j}}\left(\frac{\partial L}{\partial u_{i j}^{s}}\right)+D_{x^{j}} D_{x^{k}}\left(\frac{\partial L}{\partial u_{i j k}^{s}}\right)-\cdots\right] \\
& +D_{x^{j}}\left(W^{s}\right) \\
& \times\left[\frac{\partial L}{\partial u_{i j}^{s}}-D_{x^{k}}\left(\frac{\partial L}{\partial u_{i j k}^{s}}\right)+D_{x^{k}} D_{x^{r}}\left(\frac{\partial L}{\partial u_{i j k r}^{s}}\right)-\cdots\right] \\
& +D_{x^{j}} D_{x^{k}}\left(W^{s}\right)\left[\frac{\partial L}{\partial u_{i j k}^{s}}-D_{x^{r}}\left(\frac{\partial L}{\partial u_{i j k r}^{s}}\right)+\cdots\right]+\cdots,
\end{aligned}
$$

with

$$
W^{s}=\eta^{s}-\xi^{i} u_{i}^{s}, \quad s=1, \ldots, m
$$

3.2. Conservation Laws for (1). To search for conservation laws of (1) by Theorem 1, adjoint equation and formal Lagrangian of (1) must be known. We first construct its adjoint equation. Following the idea in [19], the adjoint equation of (1) is

$$
E_{1}^{*} \equiv v_{x t}+6 u v_{x x}+v_{x x x x}-e(t) v_{x}+n(t) v_{y y}=0,
$$

where $v$ is a new dependent variable with respect to $x, y$, and $t$.

According to the method of constructing Lagrangian in [19], the formal Lagrangian for the system consisting of (1) and (28) is

$$
L=v\left(u_{x t}+6 u_{x}^{2}+6 u u_{x x}+u_{x x x x}+e(t) u_{x}+n(t) u_{y y}\right) .
$$

By means of the symmetries of (1), conservation laws of the system consisting of (1) and (28) can be derived by
Theorem 1. However, we are only interested in the conservation laws of (1). Therefore one has to eliminate the nonlocal variable $v$ which is introduced in the adjoint equation. To solve this problem, the concepts of self-adjointness, quasiself-adjointness, and nonlinear self-adjointness are developed [20-24]. In the following, we will discuss the adjointness and nonlinear adjointness using these definitions.

Equation (1) is said to be self-adjoint if the equation obtained from the adjoint equation (28) by the substitution $v=u$ is identical with the original equation (1). It is easy to see that (28) is not identical with (1) when $v=u$, so (1) is not a self-adjoint equation. According to the definition of nonlinear self-adjointness [24], (1) is said to be nonlinearly self-adjoint if its adjoint equation (28) is satisfied for all solutions $u$ of (1) upon a substitution

$$
v=H(x, y, t, u), \quad H(x, y, t, u) \neq 0 .
$$

In other words, (1) is nonlinearly self-adjoint if and only if

$$
\left.E_{1}^{*}\right|_{v=H(x, y, t, u)}=\lambda\left(x, y, t, u, u_{x}, u_{y}, u_{t}, u_{x x}, \ldots\right) E_{1} \text {, }
$$

where $\lambda$ is an undetermined and smooth function.

From (31), we can get the following equation:

$$
\begin{aligned}
\left(H_{u}\right. & -\lambda) u_{x x x x}+n(t)\left(H_{u}-\lambda\right) u_{y y}+\left(H_{u}-\lambda\right) u_{x t} \\
& +4 H_{u u} u_{x} u_{x x x}+4 H_{x u} u_{x x x}+2 n(t) H_{y u} u_{y} \\
& +u_{x}^{2}\left(6 u H_{u u}+6 H_{u u u} u_{x x}-6 \lambda+6 H_{x x u u}\right) \\
& +u\left(12 u_{x} H_{x u}+6 H_{u} u_{x x}-6 \lambda u_{x x}+6 H_{x x}\right)+H_{t u} u_{x} \\
& -\lambda e(t) u_{x}-e(t) u_{x} H_{u}+n(t) u_{y}^{2} H_{u u} \\
& +12 H_{x u u} u_{x} u_{x x}+u_{x} u_{t} H_{u u}+6 H_{x x u} u_{x x}+4 H_{x x x u} u_{x} \\
& +H_{x u} u_{t}+4 H_{x u u u} u_{x}^{3}+3 H_{u u} u_{x x}^{2}+H_{u u u u} u_{x}^{4} \\
& +\left(-e(t) H_{x}+n(t) H_{y y}+H_{x t}+H_{x x x x}\right)=0 .
\end{aligned}
$$

Solving the above system with the aid of Maple, the final results read as

$$
\begin{gathered}
\lambda=0, \\
H=(a(t) y+b(t)) x-\frac{a_{t} y^{3}}{6 n(t)}-\frac{b_{t} y^{2}}{2 n(t)} \\
+\frac{e(t) a(t) y^{3}}{6 n(t)}+\frac{e(t) b(t) y^{2}}{2 n(t)}+k(t) y+l(t),
\end{gathered}
$$

where $a(t), b(t), k(t)$, and $l(t)$ are arbitrary functions. In summary, we have the following statements.

Theorem 2. The time-dependent KP equation (1) is nonlinearly self-adjoint.

In the following, we first construct the conservation laws for the system consisting of the initial equation (1) and its adjoint (28). 
For the symmetry in Case 1, the corresponding components of the conservation laws are

$$
\begin{aligned}
& X_{1}=f(t) u_{x} v_{t}+f_{t} u_{x} v-g(t) u_{x x x y} v-g(t) u_{x y} v_{x x} \\
& -f_{t} v_{x} u+f(t) u_{x t} v+g(t) u_{y} v_{x x x}+\frac{f_{t} e(t) v}{6} \\
& +g(t) u_{x x y} v_{x}+g(t) u_{y} v_{t} \\
& +f(t) u_{x} v_{x x x}+f(t) v_{x} u_{x x x} \\
& -f(t) u_{x x} v_{x x}+\frac{g_{t} y u_{x x} v_{x x}}{2 n(t)}-\frac{1}{6} f_{t} v_{x x x}-\frac{g_{t} y u_{y y} v}{2} \\
& +f(t) n(t) u_{y y} v+\frac{g_{t t} y v_{t}}{12 n(t)}+\frac{g_{t t} y v_{x x x}}{12 n(t)}+6 f(t) u_{x} v_{x} u \\
& -g(t) e(t) u_{y} v-6 g(t) u_{y} u_{x} v+6 g(t) u_{y} v_{x} u \\
& -6 g(t) u_{x y} u v-\frac{1}{6} f_{t} v_{t}-\frac{g_{t t} y e(t) v}{12 n(t)}-\frac{g_{t} y u_{x} v_{t}}{2 n(t)} \\
& -\frac{g_{t} y u_{x t} v}{2 n(t)}-\frac{g_{t t} y u_{x} v}{2 n(t)}+\frac{g_{t t} y u v_{x}}{2 n(t)}+\frac{g_{t} y n_{t} e(t) v}{12 n^{2}(t)} \\
& +\frac{g_{t} y n_{t} u_{x} v}{2 n^{2}(t)}-\frac{g_{t} y n_{t} v_{x} u}{2 n^{2}(t)}-\frac{g_{t} y n_{t} v_{t}}{12 n^{2}(t)}-\frac{g_{t} y n_{t} v_{x x x}}{12 n^{2}(t)} \\
& -\frac{3 g_{t} y u_{x} v_{x} u}{n(t)}-\frac{g_{t} y u_{x} v_{x x x}}{2 n(t)}-\frac{g_{t} y u_{x x x} v_{x}}{2 n(t)}, \\
& Y_{1}=-\frac{n(t) f_{t} v_{y}}{6}+\frac{y g_{t t} v_{y}}{12}-\frac{g_{t} y n_{t} v_{y}}{12 n(t)}-\frac{1}{2} g_{t} y u_{x} v_{y} \\
& +f(t) n(t) u_{x} v_{y}+g(t) n(t) u_{y} v_{y}-\frac{g_{t t} v}{12}+\frac{g_{t} n_{t} v}{12 n(t)} \\
& +\frac{1}{2} g_{t} u_{x} v+\frac{1}{2} g_{t} y u_{x y} v-f(t) n(t) u_{x y} v \\
& -g(t) v n(t) u_{y y} \\
& T_{1}=\frac{g_{t} y u_{x x} v}{2 n(t)}-f(t) u_{x x} v-g(t) u_{x y} v .
\end{aligned}
$$

For the symmetry in Case 2, the corresponding components of the conservation laws are

$$
\begin{aligned}
X_{2}= & -6 \frac{C_{2} m u_{t} v_{x} u}{p}-\frac{1}{6} f_{t} v_{x x x}-\frac{1}{6} f_{t} v_{t}+\frac{g_{t} y}{2 n(t)} u_{x x} v_{x x} \\
& +\frac{C_{2} m u_{t x x x} v}{p}-\frac{g_{t t} y}{2 n(t)} u_{x} v+\frac{g_{t t} y}{12 n(t)} v_{t}+\frac{g_{t t} y}{12 n(t)} v_{x x x} \\
& +\frac{C_{2} x}{3 p} u_{x} v_{t}+\frac{C_{2} x}{3 p} u_{x} v_{x x x}-\frac{3 g_{t} y}{n(t)} u_{x} v_{x} u-\frac{g_{t} y}{2 n(t)} u_{x} v_{t} \\
& +4 \frac{C_{2} y}{p} u_{y} v_{x} u+\frac{2 C_{2} y}{3 p} u_{y} v_{t}+\frac{2 C_{2} y}{3 p} u_{y} v_{x x x}
\end{aligned}
$$

$-3 C_{2} y u_{y} u_{x} v+3 C_{2} y u_{y} v_{x} u-6 \frac{C_{2} t}{p} u_{t} u_{x} v$

$+6 \frac{C_{2} t}{p} u_{t} u_{x} u+\frac{C_{2} t}{p} u_{t} v_{t}+\frac{C_{2} t}{p} u_{t} v_{x x x}+6 \frac{C_{2} m u_{t} u_{x} v}{p}$

$-\frac{C_{2} m u_{t} v_{x x x}}{p}-\frac{C_{2} x}{3 p} u_{x x} v_{x x}-\frac{2 C_{2} y}{3 p} u_{x y} v_{x x}$

$-3 C_{2} y u v u_{x y}-6 \frac{C_{2} t}{p} u_{x t} u v-\frac{C_{2} t}{p} u_{x t} v_{x x}$

$-\frac{g_{t} y p}{12 n(t)(t-m)} v_{t}-\frac{10 C_{2} u u_{x} v}{p}-4 \frac{C_{2} y u_{y} u_{x} v}{p}$

$+\frac{C_{2} x u_{x t} v}{3 p}+\frac{C_{2} x n(t) u_{y y} v}{3 p}-\frac{g_{t} y}{2 n(t)} u_{x t} v$

$+f(t) v n(t) u_{y y}+\frac{g_{t} y p}{2 n(t)(t-m)} u_{x} v$

$-\frac{g_{t} y p}{2 n(t)(t-m)} v_{x} u+\frac{g_{t t} y}{2 n(t)} v_{x} u$

$-\frac{g_{t} y p}{12 n(t)(t-m)} v_{x x x}+6 \frac{C_{2} m}{p} u_{x t} u v$

$+\frac{C_{2} m}{p} u_{x t} v_{x x}+\frac{C_{2} x}{3 p} v_{x} u_{x x x}-\frac{g_{t} y}{2 n(t)} u_{x x x} v_{x}$

$+\frac{2 C_{2} y}{3 p} u_{x x y} v_{x}+\frac{C_{2} t}{p} u_{x x t} v_{x}-\frac{2 C_{2} y u_{x x x y} v}{3 p}$

$-\frac{C_{2} t u_{x x x t} v}{p}-\frac{C_{2} m u_{t} v_{t}}{p}+g(t) u_{y} v_{x x x}$

$-f_{t} v_{x} u-g(t) u_{x x x y} v-f(t) u_{x x} v_{x x}+f(t) u_{x x x} v_{x}$

$+g(t) u_{x x y} v_{x}+f_{t} u_{x} v+f(t) u_{x} v_{x x x}-g(t) u_{x y} v_{x x}$

$+v f(t) u_{x t}+g(t) u_{y} v_{t}+f(t) u_{x} v_{t}+\frac{2 C_{2}}{3 p} u v_{t}$

$+\frac{4 C_{2}}{p} u^{2} v_{x}-\frac{g_{t} y}{2} u_{y y} v-\frac{C_{2} u_{x} v_{x x}}{p}+\frac{2 C_{2} x}{p} u_{x} v_{x} u$

$+6 g(t) u_{y} v_{x} u-6 g(t) u_{y} u_{x} v+\frac{C_{2} y}{2} u_{y} v_{x x x}$

$+\frac{C_{2} y}{2} u_{y} v_{t}+6 f(t) u_{x} v_{x} u+\frac{2 C_{2}}{3 p} u v_{x x x}$

$-\frac{C_{2} y}{2} v u_{x x x y}-\frac{5 C_{2} u_{x x x} v}{3 p}$

$+\frac{C_{2} y}{2} v_{x} u_{x x y}+\frac{4 C_{2}}{3 p} u_{x x} v_{x}-6 g(t) u_{x y} v u$

$-\frac{C_{2} y}{2} v_{x x} u_{x y}-\frac{4 C_{2} y u_{x y} u v}{p}-\frac{C_{2} m u_{x x t} v_{x}}{p}$, 


$$
\begin{aligned}
& Y_{2}=-\frac{g_{t t} v}{12}-\frac{C_{2} \operatorname{tn}(t) u_{y t} v}{p}+\frac{C_{2} m n(t) u_{y t} v}{p} \\
& -f(t) n(t) v u_{x y}+\frac{g_{t} v p}{12(t-m)}+\frac{2 C_{2} n(t) u v_{y}}{3 p} \\
& -\frac{g_{t} y p v_{y}}{12(t-m)}+\frac{C_{2} x n(t) u_{x} v_{y}}{3 p}+f(t) n(t) u_{x} v_{y} \\
& +\frac{2 C_{2} y n(t) u_{y} v_{y}}{3 p}+\frac{C_{2} y n(t)}{2} u_{y} v_{y}+g(t) n(t) u_{y} v_{y} \\
& +\frac{C_{2} \operatorname{tn}(t) u_{t} v_{y}}{p}-\frac{C_{2} m n(t) u_{t} v_{y}}{p}+\frac{g_{t}}{2} u_{x} v \\
& -\frac{4 C_{2} n(t) u_{y} v}{3 p}-\frac{C_{2} x n(t) u_{x y} v}{3 p}+\frac{g_{t t}}{12} y v_{y}+\frac{g_{t} y u_{x y} v}{2} \\
& -\frac{g_{t} y u_{x} v_{y}}{2}-\frac{1}{6} f_{t} n(t) v_{y}-\frac{C_{2} n(t) u_{y} v}{2} \\
& -g(t) v n(t) u_{y y}-\frac{2 C_{2} y v n(t)}{3 p} u_{y y}-\frac{C_{2} y v n(t)}{2} u_{y y}, \\
& T_{2}=-\frac{C_{2} u_{x} v}{p}-\frac{C_{2} x u_{x x} v}{3 p}+\frac{g_{t} y u_{x x} v}{2 n(t)}-f(t) u_{x x} v \\
& -\frac{2 C_{2} y u_{x y} v}{3 p}-\frac{C_{2} y u_{x y} v}{2}-g(t) u_{x y} v-\frac{C_{2} v t u_{x t}}{p} \\
& +\frac{C_{2} v m u_{x t}}{p}
\end{aligned}
$$

Here we should note that the coefficient function $n(t)$ in the expression of $X_{2}, Y_{2}$, and $T_{2}$ satisfies $n(t)=(t-m)^{p} C_{1}, m, p$, and $C_{1}$ are constants, and $p \neq 0, C_{1} \neq 0$.

For the symmetry in Case 3 , the corresponding components of the conservation laws are

$$
\begin{aligned}
X_{3}= & -\frac{x}{18} \tau_{t t} v_{x x x}+f(t) v_{x} u_{x x x}+g(t) v_{x} u_{x x y} \\
& +f(t) u_{x} v_{x x x}+\tau(t) u_{t} v_{t}-\tau_{t} u_{x} v_{x x}+g(t) u_{y} v_{x x x} \\
& +g(t) u_{y} v_{t}+\frac{2}{3} \tau_{t} u v_{t}-\tau(t) u_{x t} v_{x x}+\frac{2}{3} \tau_{t} u v_{x x x} \\
& +\tau(t) v_{x} u_{t x x}-\frac{5}{3} \tau_{t} v u_{x x x}-\tau(t) v u_{t x x x}+4 \tau_{t} u^{2} v_{x} \\
& -f_{t} v_{x} u-g(t) u_{x y} v_{x x}+\frac{1}{3} \tau_{t t} u v+f_{t} u_{x} v \\
& -f(t) u_{x x} v_{x x}-\frac{1}{6} f_{t} v_{x x x}-g(t) v u_{x x x y}+f(t) v u_{t x} \\
& +f(t) v_{t} u_{x}+\tau(t) u_{t} v_{x x x}-\frac{x}{18} \tau_{t t} v_{t}+\frac{y^{2}}{6 n} \tau_{t t t} u v_{x} \\
& +\frac{y}{2 n} g_{t t} u v_{x}-\frac{y^{2}}{6 n} \tau_{t t} v u_{x t}-\frac{1}{6} f_{t} v_{t}+2 x \tau_{t} u_{x} v_{x} u
\end{aligned}
$$

$$
\begin{aligned}
& +\frac{1}{18} \tau_{t t} v_{x x}-\frac{y^{2}}{6 n} \tau_{t t t} u_{x} v+\frac{x}{3} n \tau_{t} v u_{y y}-\frac{y}{2 n} g_{t t} u_{x} v \\
& +\frac{x}{3} \tau_{t} v u_{x t}-\frac{y^{2}}{6} \tau_{t t} v u_{y y}-\frac{y}{2} g_{t} v u_{y y}+f(t) v n u_{y y} \\
& -10 \tau_{t} u u_{x} v+\frac{x}{3} \tau_{t t} u_{x} v-\frac{x}{3} \tau_{t t} v_{x} u+\frac{y^{2}}{36 n} \tau_{t t t} v_{t} \\
& +\frac{y^{2}}{36 n} \tau_{t t t} v_{x x x}+\frac{y}{12 n} g_{t t} v_{t}+\frac{y}{12 n} g_{t t} v_{x x x}+\frac{x}{3} \tau_{t} u_{x} v_{t} \\
& +\frac{x}{3} \tau_{t} u_{x} v_{x x x}+6 f(t) u_{x} v_{x} u+\frac{2 y}{3} \tau_{t} u_{y} v_{t} \\
& +\frac{2 y}{3} \tau_{t} u_{y} v_{x x x}-6 g(t) u_{x} u_{y} v+6 g(t) v_{x} u_{y} u \\
& -6 \tau(t) u_{t} u_{x} v+6 \tau(t) u_{t} v_{x} u-\frac{x}{3} \tau_{t} u_{x x} v_{x x} \\
& -\frac{2 y}{3} \tau_{t} u_{x y} v_{x x}-6 g(t) u_{x y} u v-6 \tau(t) u_{x t} u v \\
& +\frac{x}{3} \tau_{t} u_{x x x} v_{x}+\frac{2 y}{3} \tau_{t} u_{x x y} v_{x}-\frac{2 y}{3} \tau_{t} u_{x x x y} v \\
& -\frac{y}{2 n} g_{t} u_{t x} v-\frac{y^{2}}{6 n} \tau_{t t} v_{x} u_{x x x}-\frac{y}{2 n} g_{t} u_{x x x} v_{x} \\
& -\frac{y^{2}}{n} \tau_{t t} u_{x} v_{x} u-\frac{y^{2}}{6 n} \tau_{t t} u_{x} v_{t}-\frac{y^{2}}{6 n} \tau_{t t} u_{x} v_{x x x} \\
& -\frac{3 y}{n} g_{t} u_{x} v_{x} u-\frac{y}{2 n} g_{t} u_{x} v_{t}-\frac{y}{2 n} g_{t} u_{x} v_{x x x} \\
& -4 y \tau_{t} u_{y} u_{x} v+4 y \tau_{t} u_{y} v_{x} u+\frac{y^{2}}{6 n} \tau_{t t} u_{x x} v_{x x} \\
& +\frac{y}{2 n} g_{t} u_{x x} v_{x x}-4 y \tau_{t} u_{x y} u v+\frac{4}{3} \tau_{t} u_{x x} v_{x} \\
& Y_{3}=\frac{1}{2} g_{t} v u_{x}+\frac{y}{12} g_{t t} v_{y}-\frac{y}{18} \tau_{t t t} v+\frac{y^{2}}{36} \tau_{t t t} v_{y} \\
& -\frac{1}{12} g_{t t} v+\frac{x}{3} n \tau_{t} u_{x} v_{y}+\frac{y}{3} \tau_{t t} u_{x} v+\frac{y^{2}}{6} \tau_{t t} u_{x y} v \\
& +\frac{2}{3} n \tau_{t} u v_{y}-\frac{x}{18} n \tau_{t t} v_{y}+n f(t) u_{x} v_{y}+g(t) n v_{y} u_{y} \\
& +n \tau(t) u_{t} v_{y}-\frac{4}{3} n \tau_{t} v u_{y}-n f(t) v u_{x y}-\tau(t) n v u_{t y} \\
& -\frac{y^{2}}{6} \tau_{t t} u_{x} v_{y}-\frac{y}{2} g_{t} u_{x} v_{y}+\frac{y}{2} g_{t} v u_{x y}-\frac{1}{6} n f_{t} v_{y} \\
& +\frac{2 y}{3} n \tau_{t} u_{y} v_{y}-\frac{x}{3} n \tau_{t} v u_{x y}-\frac{2 y}{3} \tau_{t} v n u_{y y} \\
& -g(t) v n u_{y y},
\end{aligned}
$$




$$
\begin{aligned}
T_{3}= & -\tau(t) v u_{x t}-\tau_{t} v u_{x}+\frac{1}{18} \tau_{t t} v-\frac{x}{3} \tau_{t} v u_{x x} \\
& +\frac{y^{2}}{6 n} \tau_{t t} u_{x x} v+\frac{y}{2 n} g_{t} u_{x x} v-f(t) v u_{x x} \\
& -\frac{2 y}{3} \tau_{t} u_{x y} v-\tau(t) v u_{x y} .
\end{aligned}
$$

For the fourth symmetry, the two functions $\tau(t)$ and $n(t)$ are determined by the differential equation (19) and they have many explicit solutions. For simplicity, we take $\tau(t)=1$; then $n(t)=1+\tan ^{2} t$ and $e(t)=(-\tan t / 2)+C_{3}$. When $f(t)=$ $g(t)=0$, the corresponding Lie symmetry is

$$
V=-\frac{y^{2}}{4} \frac{\partial}{\partial x}+y \tan t \frac{\partial}{\partial y}+\frac{\partial}{\partial t}+0 \frac{\partial}{\partial u}
$$

and the components of the conservation laws are

$$
\begin{aligned}
& X_{4}=-v u_{t x x x}-\frac{y^{2}}{4} u_{x x x} v_{x}-6 u v u_{x t}+\frac{y^{2}}{4} u_{x x} v_{x x} \\
& +6 u u_{t} v_{x}-6 v u_{t} u_{x}-C_{3} u_{t} v+v_{x} u_{t x x}+u_{t} v_{t} \\
& +\frac{\tan t}{2} u_{t} v-\frac{y^{2}}{4} u_{x} v_{x x x}-\frac{y^{2}}{4} u_{x} v_{t}-\frac{y^{2}}{4} u_{y y} v \\
& -\frac{y^{2}}{4} v u_{x t}+u_{t} v_{x x x}-\frac{3 y^{2}}{2} u_{x} v_{x} u+\frac{\tan ^{2} t}{2} y v u_{y} \\
& +y u_{y} v_{x x x} \tan t+y u_{y} v_{t} \tan t+y v_{x} u_{x x y} \tan t \\
& -6 y v u_{y} u_{x} \tan t-C_{3} y v u_{y} \tan t-\frac{\tan ^{2} t}{4} y^{2} v u_{y y} \\
& -u_{x t} v_{x x}-y v_{x x} u_{x y} \tan t-y v u_{x x x y} \tan t \\
& -6 y u v u_{x y} \tan t+6 y u u_{y} v_{x} \tan t \\
& Y_{4}=-\frac{y^{2}}{4} u_{x} v_{y}-\frac{y^{2}}{4} v_{y} u_{x} \tan ^{2} t+y v_{y} u_{y} \tan t \\
& +y v_{y} u_{y} \tan ^{3} t+v_{y} u_{t}+v_{y} u_{t} \tan ^{2} t+\frac{1}{2} v y u_{x} \\
& +\frac{y^{2}}{4} v u_{x y}+\frac{y^{2}}{4} v u_{x y} \tan ^{2} t-v u_{y} \tan t-v u_{y} \tan ^{3} t \\
& -v u_{t y}-v u_{t y} \tan ^{2} t+\frac{1}{2} v y u_{x} \tan ^{2} t \\
& -y v \tan t u_{y y}-y v \tan ^{3} t u_{y y} \text {, } \\
& T_{4}=\frac{y^{2}}{4} v u_{x x}-y v u_{x y} \tan t-v u_{x t} .
\end{aligned}
$$

We should mention that in the above components of the conservation laws for (1) and (28), $u$ is a solution of (1) and $v$ is a solution of the adjoint equation (28). Making use of the explicit solutions of (28), local conservation laws for (1) can be obtained. For example, when $a(t)=0$ and $b(t)=0$ in (34),

$$
v=k(t) y+l(t),
$$

where $k(t)$ and $l(t)$ are arbitrary functions, is an exact solution of (28). Substituting (40) into the above four conservation laws, we can obtain time-dependent and local conservation laws for (1). Here we take $\left(X_{4}, Y_{4}, T_{4}\right)$ as an illustrative example; when $v=k(t) y+l(t)$, the components of the conservation laws $\left(X_{4}, Y_{4}, T_{4}\right)$ become

$$
\begin{aligned}
& \bar{X}_{4}=-C_{3} y^{2} u_{y} k(t) \tan t-C_{3} l(t) y u_{y} \tan t \\
& -6 k(t) y^{2} u_{y} u_{x} \tan t-6 l(t) y u_{y} u_{x} \tan t+l^{\prime}(t) u_{t} \\
& -l(t) u_{x x x t}-6 k(t) y^{2} u_{x y} u \tan t-\frac{y^{3}}{4} k(t) u_{y y} \\
& -\frac{y^{2}}{4} l^{\prime}(t) u_{x}-C_{3} l(t) u_{t}+\frac{1}{2} l(t) u_{t} \tan t-6 l(t) u_{t} u_{x} \\
& -6 l(t) u_{x t} u-\frac{y^{2}}{4} l(t) u_{y y}-k(t) y u_{x x x t}-\frac{y^{3}}{4} k^{\prime}(t) u_{x} \\
& +k^{\prime}(t) y u_{t}-\frac{y^{2}}{4} l(t) u_{x t}-\frac{y^{3}}{4} k(t) u_{x t} \\
& -y l(t) u_{x x x y} \tan t-k(t) y^{2} u_{x x x y} \tan t \\
& -\frac{y^{3}}{4} k(t) u_{y y} \tan ^{2} t+\frac{y}{2} k(t) u_{t} \tan t+l^{\prime}(t) y u_{y} \tan t \\
& -6 k(t) y u u_{x t}+\frac{y}{2} l(t) u_{y} \tan ^{2} t+\frac{y^{2}}{2} k(t) u_{y} \tan ^{2} t \\
& -6 k(t) y u_{t} u_{x}-C_{3} k(t) y u_{t}-\frac{y^{2} \tan ^{2} t}{4} l(t) u_{y y} \\
& +k^{\prime}(t) y^{2} u_{y} \tan t-6 l(t) y u_{x y} u \tan t \\
& \bar{Y}_{4}=-l(t) y u_{y y} \tan ^{3} t-l(t) y u_{y y} \tan t-l(t) u_{y t} t+k(t) u_{t} \\
& -k(t) y^{2} \tan ^{3} t u_{y y}-k(t) y \tan ^{2} t u_{y t}+\frac{y}{2} l(t) u_{x} \tan ^{2} t \\
& +\frac{y^{2}}{4} l(t) u_{x y} \tan ^{2} t+\frac{y^{3}}{4} k(t) u_{x y}+k(t) u_{t} \tan ^{2} t \\
& +\frac{y^{2}}{4} k(t) u_{x}-l(t) u_{y t} \tan ^{2} t-y k(t) u_{y t}-l(t) u_{y} \tan ^{3} t \\
& -l(t) u_{y} \tan t+\frac{y^{2}}{4} l(t) u_{x y}+\frac{y}{2} l(t) u_{x} \\
& +\frac{y^{2}}{4} k(t) u_{x} \tan ^{2} t+\frac{y^{3}}{4} k(t) u_{x y} \tan ^{2} t \\
& -k(t) y^{2} u_{y y} \tan t
\end{aligned}
$$$$
\bar{T}_{4}=\frac{1}{4}(k(t) y+l(t))\left(y^{2} u_{x x}-4 y u_{x y} \tan t-4 u_{x t}\right) .
$$ 
These are local and explicit conservation laws of (1). Next we show that the above conservation laws $\left(\bar{X}_{4}, \bar{Y}_{4}, \bar{T}_{4}\right)$ are nontrivial:

$$
\begin{aligned}
& D_{x}\left(\bar{X}_{4}\right)+D_{y}\left(\bar{Y}_{4}\right)+D_{t}\left(\bar{T}_{4}\right) \\
& =-C_{3} y^{2} k(t) u_{x y} \tan t-l(t) u_{x x x x t}-l(t) u_{x t t} \\
& -k(t) y u_{x t t}-12 l(t) u_{x} u_{x t}-2 l(t) u_{y y} \tan ^{3} t \\
& +\frac{1}{2} l(t) u_{x} \tan ^{2} t-2 l(t) u_{y y} \tan t+\frac{1}{2} y k(t) u_{x} \\
& -k(t) y u_{x x x x t}-6 l(t) u u_{x x t}-6 l(t) u_{t} u_{x x} \\
& +\frac{1}{2} l(t) u_{x t} \tan t-C_{3} l(t) u_{x t}-l(t) u_{y y t} \tan ^{2} t \\
& -k(t) y u_{y y t}-6 y^{2} k(t) u u_{x x y} \tan t \\
& -12 y^{2} k(t) u_{x} u_{x y} \tan t-12 y l(t) u_{x} u_{x y} \tan t \\
& -6 l(t) y u u_{x x y} \tan t-6 k(t) y^{2} u_{y} u_{x x} \tan t \\
& -6 l(t) y u_{y} u_{x x} \tan t-l(t) u_{y y t}-C_{3} l(t) y u_{x y} \tan t \\
& +\frac{1}{2} l(t) u_{x}-k(t) y u_{y y t} \tan ^{2} t-l(t) y u_{y y y} \tan ^{3} t \\
& +\frac{1}{2} y l(t) u_{x y} \tan ^{2} t-C_{3} k(t) y u_{x t}-6 y k(t) u_{t} u_{x x} \\
& +\frac{1}{2} y^{2} k(t) u_{x y} \tan ^{2} t-l(t) y u_{x x x x y} \tan t \\
& -12 k(t) y u_{x} u_{x t}-6 k(t) y u u_{x x t}+\frac{1}{2} y k(t) u_{x t} \tan t \\
& -k(t) y^{2} u_{x x x x y} \tan t-k(t) y^{2} u_{x y t} \tan t \\
& -l(t) y u_{x y t} \tan t-2 k(t) y u_{y y} \tan ^{3} t \\
& +\frac{1}{2} y k(t) u_{x} \tan ^{2} t-2 k(t) y u_{y y} \tan t \\
& -k(t) y^{2} u_{y y y} \tan t-l(t) y u_{y y y} \tan t \\
& -k(t) y^{2} u_{y y y} \tan ^{3} t
\end{aligned}
$$

Obviously, if $k(t), l(t)$ are not zero at the same time, $D_{x}\left(\bar{X}_{4}\right)+$ $D_{y}\left(\bar{Y}_{4}\right)+D_{t}\left(\bar{T}_{4}\right) \neq 0$. And we can easily check that

$$
\begin{aligned}
& \left(D_{x}\left(X_{4}\right)+D_{y}\left(Y_{4}\right)\right. \\
& \left.\quad+D_{t}\left(T_{4}\right)\right)\left.\right|_{u_{x x x x}=-u_{x t}-6 u_{x}{ }^{2}-6 u u_{x x}-e(t) u_{x}-n(t) u_{y y}} \equiv 0 .
\end{aligned}
$$

\section{Symmetry Reductions and New Exact Solutions of (1)}

In Section 2, we obtain the Lie symmetries of (1). In this section, we will investigate the symmetry reductions and exact solutions for the equation. Using the obtained symmetries (3), similarity variables and symmetry reductions can be found by solving the corresponding characteristic equation:

$$
\frac{d x}{\xi}=\frac{d y}{\eta}=\frac{d t}{\tau}=\frac{d u}{\phi} .
$$

For the four different cases, we determine the following symmetry reductions and exact solutions of (1).

4.1. For the Symmetry in Case 1 , Where e $(t)$ and $n(t)(n(t) \neq 0)$ Are Arbitrary Functions.

(i) When $g(t)=0, f(t) \neq 0$, we can obtain

$$
u=\frac{f_{t} x}{6 f}+\Omega(y, t)
$$

and $\Omega(y, t)$ is a solution of the following reduction equation:

$$
\frac{f_{t t}}{6 f}+\frac{e f_{t}}{6 f}+n \Omega_{y y}=0 .
$$

From the above equation, we can obtain an algebraically explicit analytical solution for (1):

$$
u=\frac{f_{t} x}{6 f}-\frac{f_{t t}+e f_{t}}{12 n f} y^{2}+F_{1}(t) y+F_{2}(t)
$$

where $F_{1}(t)$ and $F_{2}(t)$ are arbitrary functions of $t$.

(ii) When $f(t)=0, g(t)=t$, the corresponding symmetry is

$$
V=-\frac{y}{2 n} \frac{\partial}{\partial x}+t \frac{\partial}{\partial y}+0 \frac{\partial}{\partial t}+\frac{n_{t}}{12 n^{2}} y \frac{\partial}{\partial u}
$$

By the characteristic equations of the symmetry, we have $u=\Omega(\theta, t), \theta=y^{2} / 2+2 n x t$. Substituting it into (1), we get a symmetry reduction of (1):

$$
\begin{aligned}
\Omega_{\theta t} & +\frac{\theta}{t} \Omega_{\theta \theta}+12 n t\left(\Omega_{\theta} \Omega\right)_{\theta}+8 n^{3} t^{3} \Omega_{\theta \theta \theta \theta} \\
+ & \left(\frac{3}{2 t}-\frac{n_{t}}{n}+e(t)\right) \Omega_{\theta}+\frac{n_{t}^{2}}{6 n^{3} t}-\frac{n_{t t}}{12 n^{2} t} \\
- & \frac{e(t) n_{t}}{12 n^{2} t}=0 .
\end{aligned}
$$

If the coefficient functions $e(t)=0, n(t)=$ Const., the obtained symmetry reduction can be simplified to

$$
\Omega_{\theta t}+\frac{\theta}{t} \Omega_{\theta \theta}+\frac{3}{2 t} \Omega_{\theta}+12 n t\left(\Omega_{\theta} \Omega\right)_{\theta}+8 n^{3} t^{3} \Omega_{\theta \theta \theta \theta}=0 .
$$

Integrating (50) with respect to $\theta$ and taking the constant of integration to zero, we get the following equation:

$$
\Omega_{t}+12 n t \Omega_{\theta} \Omega+8 n^{3} t^{3} \Omega_{\theta \theta \theta}+\frac{\theta}{t} \Omega_{\theta}+\frac{1}{2 t} \Omega=0 .
$$


Equation (51) is the $(1+1)$-dimensional generalized $\mathrm{KdV}$ equation with variable coefficients. To the best of our knowledge, exact solutions of (51) have not been studied up to now. Solving (51) by the method in [25], we can get the following solutions for (1):

$$
\begin{gathered}
u=\Omega(\theta, t)=\frac{\theta}{24 n t^{2}}+\frac{M_{3}}{24 n t M_{1}} \\
-\frac{8 n^{2} M_{1}^{2} c_{2}}{3 t}-\frac{8 n^{2} M_{1}^{2} c_{4}}{t} P^{2}(\varphi), \\
\varphi=M_{1} \theta t^{-3 / 2}+M_{3} t^{-1 / 2}+M_{2},
\end{gathered}
$$

where $M_{1}, M_{2}$, and $M_{3}$ are arbitrary constants and the function $P(\varphi)$ satisfies

$$
P^{\prime 2}=c_{0}+c_{2} P^{2}+c_{4} P^{4}
$$

where $c_{0}, c_{2}$, and $c_{4}$ are constants; solutions of (53) have been given in [26]. By means of the solutions of (53), plenty of solutions for (1) can be obtained; for example,

$$
\begin{aligned}
u_{1}= & \frac{y^{2} / 2+2 n x t}{24 n t^{2}}+\frac{M_{3}}{24 n t M_{1}} \\
& -\frac{8 n^{2} M_{1}^{2}\left(-k^{2}-1\right)}{3 t}-\frac{8 n^{2} M_{1}^{2} k^{2} n^{2}(\varphi)}{t}, \\
u_{2}= & \frac{y^{2} / 2+2 n x t}{24 n t^{2}}+\frac{M_{3}}{24 n t M_{1}} \\
& -\frac{8 n^{2} M_{1}^{2}\left(-k^{2}-1\right)}{3 t}-\frac{8 n^{2} M_{1}^{2} n s^{2}(\varphi)}{t}, \\
u_{3}= & \left.\frac{y^{2} / 2+2 n x t}{24 n t^{2}}+\frac{c_{3}}{24 n t M_{1}}-\frac{8 n^{2} M_{1}^{2} c_{2}}{3 t}, k^{2}, c_{2}=-1-k^{2}, c_{4}=1\right), \\
& +\frac{8 n^{2} M_{1}^{2} c_{2} \operatorname{sech}^{2}(\varphi)}{t}, \quad\left(c_{0}=0, c_{2}>0, c_{4}<0\right), \\
u_{4}= & \frac{y^{2} / 2+2 n x t}{24 n t^{2}}+\frac{M_{3}}{24 n t M_{1}}-\frac{8 n^{2} M_{1}^{2} c_{2}}{3 t} \\
& \left.+c_{1}^{2} c_{2} \tanh ^{2}(\varphi) \frac{c_{2}^{2}}{4 c_{4}}, c_{2}<0, c_{4}>0\right),
\end{aligned}
$$

where $k(0<k<1)$ denotes the modulus of the Jacobi elliptic function.

(iii) When $e(t)=0, n(t)=(t-m)^{p} C_{1}, p \neq 0, C_{1} \neq 0, f(t)=$ $M_{0}$, and $g(t)=1$, we can get

$$
u=\Omega(\theta, t), \quad \theta=x-M_{0} y
$$

And $\Omega(\theta, t)$ satisfies the following reduction equation:

$$
\begin{gathered}
\Omega_{\theta t}+6\left(\Omega_{\theta}^{2}+\Omega \Omega_{\theta \theta}\right)+\Omega_{\theta \theta \theta \theta} \\
+M_{0}^{2} C_{1}(t-m)^{p} \Omega_{\theta \theta}=0 .
\end{gathered}
$$

The above equation can be integrated by $\theta$ and, when we take the constant of integration to zero, we get a reduced reduction equation:

$$
\Omega_{t}+6 \Omega \Omega_{\theta}+\Omega_{\theta \theta \theta}+M_{0}^{2} C_{1}(t-m)^{p} \Omega_{\theta}=0
$$

Equation (57) is variable coefficient $\mathrm{KdV}$ equation and soliton-like solutions have been obtained in [27]. By means of the known solutions, many explicit solutions of (1) can be obtained. For example,

$$
\begin{gathered}
u_{1}=k_{1}+2 c k_{4}^{2} \operatorname{sech}^{2}(\sqrt{c} \varphi), \\
\varphi=k_{4}\left(x-M_{0} y\right)-6 k_{1} k_{4} t-4 c k_{4}^{3} t \\
-\frac{M_{0}^{2} C_{1} k_{4}}{p+1}(t-m)^{p+1}, \\
u_{2}=k_{1}-2 c k_{4}^{2} \tanh ^{2}(\varphi), \\
\varphi=k_{4}\left(x-M_{0} y\right)-6 k_{1} k_{4} t+8 k_{4}^{3} t \\
-\frac{M_{0}^{2} C_{1} k_{4}}{p+1}(t-m)^{p+1},
\end{gathered}
$$

where $k_{1}, k_{4}$, and $c$ are constants.

(iv) When $e(t) \neq 0$ and $n(t)=N_{0} \exp \left(\left(\int\left(e_{t}-2 e^{2}\right) / e\right) d t\right)$, $f(t)=N_{1}, g(t)=1$. By the corresponding characteristic equation of the symmetry, we have

$$
u=\Omega(\theta, t), \quad \theta=x-N_{1} y .
$$

Substituting it into (1), we get the following symmetry reduction of (1):

$$
\begin{gathered}
\Omega_{\theta t}+6\left(\Omega_{\theta}^{2}+\Omega \Omega_{\theta \theta}\right)+\Omega_{\theta \theta \theta \theta}+e(t) \Omega_{\theta} \\
+N_{1}^{2} N_{0} \exp \left(\int \frac{e_{t}-2 e^{2}}{e} d t\right) \Omega_{\theta \theta} .
\end{gathered}
$$

Integrating the above equation with respect to $\theta$ and taking the constant of integration to zero, the obtained reduction equation becomes

$$
\begin{aligned}
\Omega_{t} & +6 \Omega \Omega_{\theta}+\Omega_{\theta \theta \theta}+e(t) \Omega \\
& +N_{1}^{2} N_{0} \exp \left(\int \frac{e_{t}-2 e^{2}}{e} d t\right) \Omega_{\theta} .
\end{aligned}
$$

Equation (61) is a variable coefficient $\mathrm{KdV}$ equation [28, 29].

4.2. For the Symmetry in Case $2, e(t)=0, n(t)=(t-m)^{p} C_{1}, p \neq 0$, $C_{1} \neq 0$. When $f(t)=g(t)=0, m=0, p=C_{2}=2 / 3$, then 
$n(t)=C_{1} t^{2 / 3}$, and $C_{1} \neq 0$; the corresponding symmetry of (1) is

$$
V=\frac{x}{3} \frac{\partial}{\partial x}+y \frac{\partial}{\partial y}+t \frac{\partial}{\partial t}-\frac{2}{3} u \frac{\partial}{\partial u}
$$

By the characteristic equations of the symmetry, we can get the explicit solutions for (1)

$$
u=\Omega(\theta, \delta) t^{-2 / 3}, \quad \theta=\frac{x^{3}}{t}, \delta=\frac{y}{t},
$$

where the function $\Omega(\theta, \delta)$ satisfies the following reduction equation:

$$
\begin{aligned}
- & 3 \theta^{5 / 3} \Omega_{\theta \theta}-3 \theta^{2 / 3} \delta \Omega_{\theta \delta}-5 \theta^{2 / 3} \Omega_{\theta} \\
& +54 \theta^{4 / 3}\left(\Omega_{\theta}^{2}+\Omega_{\theta \theta} \Omega\right)+36 \theta^{1 / 3} \Omega_{\theta} \Omega \\
& +81 \theta^{8 / 3} \Omega_{\theta \theta \theta \theta}+324 \theta^{5 / 3} \Omega_{\theta \theta \theta}+180 \theta^{2 / 3} \Omega_{\theta \theta} \\
& +C_{1} \Omega_{\delta \delta}=0 .
\end{aligned}
$$

Equation (64) is difficult to solve and we will study its exact solutions in a future paper.

4.3. For the Symmetry in Case 3, $e(t)=0, n(t)=$ Const., and $\tau(t) \neq 0$. When $f(t)=0, g(t)=0$, the corresponding symmetry is

$$
\begin{aligned}
V= & \left(\frac{\tau_{t}}{3} x-\frac{\tau_{t t}}{6 n} y^{2}\right) \frac{\partial}{\partial x}+\frac{2}{3} \tau_{t} y \frac{\partial}{\partial y}+\tau(t) \frac{\partial}{\partial t} \\
& +\left(-\frac{2 \tau_{t}}{3} u+\frac{\tau_{t t}}{18} x-\frac{\tau_{t t t}}{36 n} y^{2}\right) \frac{\partial}{\partial u} .
\end{aligned}
$$

By the characteristic equation of the symmetry, we have

$$
\begin{array}{r}
u=\frac{1}{18 \tau} x \tau_{t}-\frac{1}{36 n \tau} y^{2} \tau_{t t}+\frac{1}{54 n \tau^{2}} y^{2} \tau_{t}^{2}+\Omega(\theta, \delta) \tau^{-2 / 3} \\
\theta=x \tau^{-1 / 3}+\frac{1}{6 n} y^{2} \tau_{t} \tau^{-4 / 3}, \delta=y \tau^{-2 / 3}
\end{array}
$$

Substituting it into (1), we get a symmetry reduction of (1):

$$
6 \Omega_{\theta}^{2}+6 \Omega_{\theta \theta} \Omega+\Omega_{\theta \theta \theta \theta}+n \Omega_{\delta \delta}=0 .
$$

Equation (67) is the special case of $(2+1)$-dimensional Boussinesq equation and exact solutions of (67) have been studied by Chen and Zhang in [30] (with $a=0, b=0, r=$ $-3 / n$, and $s=-1 / n)$. With the help of the known solutions in [30], many explicit solutions of (1) can be obtained. We list the following soliton solutions $\left(u_{1}-u_{4}\right)$ and Jacobi elliptic function solutions $\left(u_{5}-u_{17}\right)$ :

$$
\begin{aligned}
& u_{1}=\left(\frac{-n \omega^{2}}{6 \alpha^{2}}+\frac{4}{3} \alpha^{2}\right) \tau^{-2 / 3}-2 \alpha^{2} \tau^{-2 / 3} \tanh ^{2}(\varphi) \\
& +\frac{\tau_{t} x}{18 \tau}-\frac{\tau_{t t} y^{2}}{36 n \tau}+\frac{\tau_{t}^{2} y^{2}}{54 n \tau^{2}}, \\
& u_{2}=\left(\frac{-n \omega^{2}}{6 \alpha^{2}}-\frac{2}{3} \alpha^{2}\right) \tau^{-2 / 3}+2 \alpha^{2} \tau^{-2 / 3} \operatorname{sech}^{2}(\varphi) \\
& +\frac{\tau_{t} x}{18 \tau}-\frac{\tau_{t t} y^{2}}{36 n \tau}+\frac{\tau_{t}^{2} y^{2}}{54 n \tau^{2}}, \\
& u_{3}=\left(\frac{-n \omega^{2}}{6 \alpha^{2}}+\frac{1}{3} \alpha^{2}\right) \tau^{-2 / 3} \\
& -\frac{\alpha^{2}}{2} \tau^{-2 / 3} \frac{\varepsilon \tanh ^{4}(\varphi)+\beta(1+\operatorname{sech}(\varphi))^{4}}{\tanh ^{2}(\varphi)(1+\operatorname{sech}(\varphi))^{2}} \\
& +\frac{\tau_{t} x}{18 \tau}-\frac{\tau_{t t} y^{2}}{36 n \tau}+\frac{\tau_{t}^{2} y^{2}}{54 n \tau^{2}} \\
& u_{4}=\left(\frac{-n \omega^{2}}{6 \alpha^{2}}-\frac{2}{3} \alpha^{2}\right) \tau^{-2 / 3}-2 \alpha^{2} \tau^{-2 / 3} \frac{\operatorname{sech}^{2}(\varphi)}{\tanh ^{2}(\varphi)} \\
& +\frac{\tau_{t} x}{18 \tau}-\frac{\tau_{t t} y^{2}}{36 n \tau}+\frac{\tau_{t}^{2} y^{2}}{54 n \tau^{2}}, \\
& u_{5}=\left(\frac{-n \omega^{2}}{6 \alpha^{2}}+\frac{2}{3} \alpha^{2}+\frac{2}{3} \alpha^{2} m^{2}\right) \tau^{-2 / 3}-2 \alpha^{2} \tau^{-2 / 3} \\
& \times \frac{\varepsilon+\beta m^{2} \operatorname{sn}^{4}(\varphi)}{\operatorname{sn}^{2}(\varphi)}+\frac{\tau_{t} x}{18 \tau}-\frac{\tau_{t t} y^{2}}{36 n \tau}+\frac{\tau_{t}^{2} y^{2}}{54 n \tau^{2}},
\end{aligned}
$$

$$
\begin{aligned}
u_{6}= & \left(\frac{-n \omega^{2}}{6 \alpha^{2}}+\frac{2}{3} \alpha^{2}+\frac{2}{3} \alpha^{2} m^{2}\right) \tau^{-2 / 3}-2 \alpha^{2} \tau^{-2 / 3} \\
& \times \frac{\varepsilon \operatorname{dn}^{4}(\varphi)+\beta m^{2} \mathrm{cn}^{4}(\varphi)}{\operatorname{cn}^{2}(\varphi) \operatorname{dn}^{2}(\varphi)}+\frac{\tau_{t} x}{18 \tau}-\frac{\tau_{t t} y^{2}}{36 n \tau}+\frac{\tau_{t}^{2} y^{2}}{54 n \tau^{2}}
\end{aligned}
$$$$
u_{7}=\left(\frac{-n \omega^{2}}{6 \alpha^{2}}-\frac{4}{3} \alpha^{2} m^{2}+\frac{2}{3} \alpha^{2}\right) \tau^{-2 / 3}-2 \alpha^{2} \tau^{-2 / 3}
$$

$$
\begin{aligned}
& \times \frac{\varepsilon\left(1-m^{2}\right)-\beta m^{2} \mathrm{cn}^{4}(\varphi)}{\mathrm{cn}^{2}(\varphi)}+\frac{\tau_{t} x}{18 \tau}-\frac{\tau_{t t} y^{2}}{36 n \tau}+\frac{\tau_{t}^{2} y^{2}}{54 n \tau^{2}}, \\
u_{8}= & \left(\frac{-n \omega^{2}}{6 \alpha^{2}}-\frac{4}{3} \alpha^{2}+\frac{2}{3} \alpha^{2} m^{2}\right) \tau^{-2 / 3}+2 \alpha^{2} \tau^{-2 / 3} \\
& \times \frac{\varepsilon\left(1-m^{2}\right)+\beta \operatorname{dn}^{4}(\varphi)}{\operatorname{dn}^{2}(\varphi)}+\frac{\tau_{t} x}{18 \tau}-\frac{\tau_{t t} y^{2}}{36 n \tau}+\frac{\tau_{t}^{2} y^{2}}{54 n \tau^{2}},
\end{aligned}
$$




$$
\begin{aligned}
& u_{9}=\left(\frac{-n \omega^{2}}{6 \alpha^{2}}-\frac{4}{3} \alpha^{2}+\frac{2}{3} \alpha^{2} m^{2}\right) \tau^{-2 / 3} \\
& -2 \alpha^{2} \tau^{-2 / 3} \frac{\varepsilon\left(1-m^{2}\right) \operatorname{sn}^{4}(\varphi)+\beta \mathrm{cn}^{4}(\varphi)}{\operatorname{sn}^{2}(\varphi) \mathrm{cn}^{2}(\varphi)} \\
& +\frac{\tau_{t} x}{18 \tau}-\frac{\tau_{t t} y^{2}}{36 n \tau}+\frac{\tau_{t}^{2} y^{2}}{54 n \tau^{2}}, \\
& u_{10}=\left(\frac{-n \omega^{2}}{6 \alpha^{2}}-\frac{4}{3} \alpha^{2} m^{2}+\frac{2}{3} \alpha^{2}\right) \tau^{-2 / 3} \\
& -2 \alpha^{2} \tau^{-2 / 3} \frac{\varepsilon \operatorname{dn}^{4}(\varphi)-\beta m^{2}\left(1-m^{2}\right) \operatorname{sn}^{4}(\varphi)}{\operatorname{sn}^{2}(\varphi) \operatorname{dn}^{2}(\varphi)} \\
& +\frac{\tau_{t} x}{18 \tau}-\frac{\tau_{t t} y^{2}}{36 n \tau}+\frac{\tau_{t}^{2} y^{2}}{54 n \tau^{2}} \\
& u_{11}=\left(\frac{-n \omega^{2}}{6 \alpha^{2}}-\frac{1}{3} \alpha^{2}+\frac{2}{3} \alpha^{2} m^{2}\right) \tau^{-2 / 3} \\
& -\frac{\alpha^{2}}{2} \tau^{-2 / 3} \frac{\varepsilon \operatorname{sn}^{4}(\varphi)+\beta(1 \pm \mathrm{cn}(\varphi))^{4}}{\operatorname{sn}^{2}(\varphi)(1 \pm \operatorname{cn}(\varphi))^{2}} \\
& +\frac{\tau_{t} x}{18 \tau}-\frac{\tau_{t t} y^{2}}{36 n \tau}+\frac{\tau_{t}^{2} y^{2}}{54 n \tau^{2}} \\
& u_{12}=\left(\frac{-n \omega^{2}}{6 \alpha^{2}}-\frac{1}{3} \alpha^{2}+\frac{2}{3} \alpha^{2} m^{2}\right) \tau^{-2 / 3} \\
& -\frac{\alpha^{2}}{2} \tau^{-2 / 3} \frac{\varepsilon \mathrm{cn}^{4}(\varphi)+\beta\left(\sqrt{1-m^{2}} \operatorname{sn}(\varphi) \pm \operatorname{dn}(\varphi)\right)^{4}}{\operatorname{cn}^{2}(\varphi)\left(\sqrt{1-m^{2}} \operatorname{sn}(\varphi) \pm \operatorname{dn}(\varphi)\right)^{2}} \\
& +\frac{\tau_{t} x}{18 \tau}-\frac{\tau_{t t} y^{2}}{36 n \tau}+\frac{\tau_{t}^{2} y^{2}}{54 n \tau^{2}} \\
& u_{13}=\left(\frac{-n \omega^{2}}{6 \alpha^{2}}-\frac{1}{3} \alpha^{2}-\frac{1}{3} \alpha^{2} m^{2}\right) \tau^{-2 / 3} \\
& +\frac{\alpha^{2}}{2}\left(1-m^{2}\right) \tau^{-2 / 3} \frac{\varepsilon \operatorname{dn}^{4}(\varphi)+\beta(1 \pm m \operatorname{sn}(\varphi))^{4}}{\operatorname{dn}^{2}(\varphi)(1 \pm m \operatorname{sn}(\varphi))^{2}} \\
& +\frac{\tau_{t} x}{18 \tau}-\frac{\tau_{t t} y^{2}}{36 n \tau}+\frac{\tau_{t}^{2} y^{2}}{54 n \tau^{2}} \\
& u_{14}=\left(\frac{-n \omega^{2}}{6 \alpha^{2}}-\frac{1}{3} \alpha^{2}-\frac{1}{3} \alpha^{2} m^{2}\right) \tau^{-2 / 3} \\
& -\frac{\alpha^{2}}{2}\left(1-m^{2}\right) \tau^{-2 / 3} \frac{\varepsilon \mathrm{cn}^{4}(\varphi)+\beta(1 \pm \operatorname{sn}(\varphi))^{4}}{\mathrm{cn}^{2}(\varphi)(1 \pm \operatorname{sn}(\varphi))^{2}} \\
& +\frac{\tau_{t} x}{18 \tau}-\frac{\tau_{t t} y^{2}}{36 n \tau}+\frac{\tau_{t}^{2} y^{2}}{54 n \tau^{2}} \\
& u_{15}=\left(\frac{-n \omega^{2}}{6 \alpha^{2}}-\frac{1}{3} \alpha^{2}-\frac{1}{3} \alpha^{2} m^{2}\right) \tau^{-2 / 3}
\end{aligned}
$$

where $\varphi=\alpha\left(x \tau^{-1 / 3}+(1 / 6 n) y^{2} \tau_{t} \tau^{-4 / 3}\right)+\omega\left(y \tau^{-2 / 3}\right), \alpha$ and $\omega$ are constants, $k(0<k<1)$ denotes the modulus of the Jacobi elliptic function, and $\varepsilon$ and $\beta$ are arbitrary elements of $\{0,1\}$. We should mention that the soliton solution $u_{1}$ is the limit of $u_{5}$ when $m \rightarrow 1, \varepsilon=0, \beta=1$. The solutions $u_{2}, u_{3}$, and $u_{4}$ are the limit of $u_{7}, u_{11}$, and $u_{9}$, respectively, when $m \rightarrow 1$, $\beta=1$.

4.4. For the Symmetry in Case $4, e(t)=-n_{t} / 4 n+C_{3} / \tau(t), n(t)$, and $\tau(t)$ Satisfy (19). For simplicity, we take $f(t)=g(t)=0$, $\tau(t)=1$; then $n(t)=1+\tan ^{2} t$ and $e(t)=-\tan t / 2+C_{3}$. Solving the corresponding characteristic equation, we get

$$
u=\Omega(\theta, \delta), \quad \theta=x+\frac{y^{2}}{4} \sin t \cos t, \delta=y \cos t
$$

Substituting it into (1), we get a symmetry reduction of (1):

$$
\frac{\delta^{2}}{4} \Omega_{\theta \theta}+6 \Omega_{\theta \theta} \Omega+6 \Omega_{\theta}^{2}+\Omega_{\theta \theta \theta \theta}+C_{3} \Omega_{\theta}+\Omega_{\delta \delta}=0 .
$$

Obviously, $\Omega=-\left(C_{3} / 6\right) \theta+N_{1} \delta+N_{2}$ is a solution of (70). From that, we can get an algebraically explicit analytical solution for (1) as follows:

$$
u=-\frac{C_{3}}{6}\left(x+\frac{y^{2}}{4} \sin t \cos t\right)+N_{1} y \cos t+N_{2},
$$

where $N_{1}$ and $N_{2}$ are integral constants. And, if $C_{3}=0$, (70) becomes the following $(2+1)$-dimensional variable coefficient Boussinesq equation:

$$
\frac{\delta^{2}}{4} \Omega_{\theta \theta}+6 \Omega_{\theta \theta} \Omega+6 \Omega_{\theta}^{2}+\Omega_{\theta \theta \theta \theta}+\Omega_{\delta \delta}=0 .
$$


Remark 3. To the best of our knowledge, the symmetry reductions obtained in this paper have not been reported in the existent literature, so they are completely new. The exact solutions of (1) obtained here are all different from the known solutions and they are also new. All the solutions and conservation laws obtained in this paper for (1) have been checked by Maple software.

\section{Conclusions}

In summary, by performing Lie symmetry analysis to (1), four cases of geometric symmetries are obtained when the coefficient functions satisfy four different constraint conditions. According to the relationship between symmetry and conservation laws given by Ibragimov, many explicit and nontrivial conservation laws, which includes arbitrary functions of $t$, are derived. These conservation laws may be useful for the explanation of some practical physical problems. Using the associated vector fields of the obtained symmetry, $(1)$ is reduced to $(1+1)$-dimensional nonlinear partial differential equations including different types of variable coefficient KdV equation (see (51), (57), and (61)), special case of $(2+1)$-dimensional Boussinesq equation (see (67) and (72)), and other reduction equations (see (64) and (70)). Many new explicit solutions of (1) have been derived by solving the reduction equations. These solutions, including soliton solutions, Jacobi doubly periodic solutions, and algebraically explicit analytical solutions, can make one discuss the behavior of solutions and also provide mathematical foundation for the explanation of some interesting physical phenomena.

\section{Conflict of Interests}

The author declares that there is no conflict of interests regarding the publication of this paper.

\section{Acknowledgment}

The work is supported by the Natural Science Foundation of Shandong Province (ZR2013AQ005 and ZR2010AL019).

\section{References}

[1] G. W. Bluman and S. Kumei, Symmetries and Differential Equations, vol. 81 of Applied Mathematical Sciences, Springer, New York, NY, USA, 1989.

[2] G. W. Bluman and S. C. Anco, Symmetry and Integration Methods for Differential Equations, vol. 154 of Applied Mathematical Sciences, Springer, New York, NY, USA, 2002.

[3] N. H. Ibragimov, Transformation Groups Applied to Mathematical Physics, Mathematics and its Applications (Soviet Series), D. Reidel Publishing, Dordrecht, The Netherlands, 1985.

[4] P. J. Olver, Applications of Lie Groups to Differential Equations, vol. 107 of Graduate Texts in Mathematics, Springer, New York, NY, USA, 1986.

[5] P. D. Lax, "Integrals of nonlinear equations of evolution and solitary waves," Communications on Pure and Applied Mathematics, vol. 21, pp. 467-490, 1968.
[6] T. B. Benjamin, “The stability of solitary waves," Proceedings of the Royal Society A, vol. 328, pp. 153-183, 1972.

[7] M. J. Ablowitz and P. A. Clarkson, Solitons, Nonlinear Evolution Equations and Inverse Scattering, vol. 149 of London Mathematical Society Lecture Note Series, Cambridge University Press, Cambridge, UK, 1991.

[8] R. Hirota, "Exact solution of the korteweg-de vries equation for multiple Collisions of solitons," Physical Review Letters, vol. 27, no. 18, pp. 1192-1194, 1971.

[9] J. Weiss, M. Tabor, and G. Carnevale, "The Painlevé property for partial differential equations," Journal of Mathematical Physics, vol. 24, no. 3, pp. 522-526, 1983.

[10] W. Hereman and W. Zhuang, "Symbolic software for soliton theory," Acta Applicandae Mathematicae, vol. 39, no. 1-3, pp. 361-378, 1995.

[11] C. Zhang, B. Tian, L.-L. Li, and T. Xu, "Analytic analysis on a generalized $(2+1)$-dimensional variable-coefficient Kortewegde Vries equation using symbolic computation," International Journal of Modern Physics B, vol. 24, no. 27, pp. 5359-5370, 2010.

[12] E. Yomba, "Construction of new soliton-like solutions for the $(2+1)$ dimensional KdV equation with variable coefficients," Chaos, Solitons and Fractals, vol. 21, no. 1, pp. 75-79, 2004.

[13] E. Yomba, "Abundant families of Jacobi elliptic function-like solutions for a generalized variable coefficients $2 \mathrm{D} \mathrm{KdV}$ equation via the extended mapping method," Physics Letters A, vol. 349, no. 1-4, pp. 212-219, 2006.

[14] S. Shen, J. Zhang, C. Ye, and Z. Pan, "New exact solutions of the $(2+1)$-dimensional KdV equation with variable coefficients," Physics Letters A, vol. 337, no. 1-2, pp. 101-106, 2005.

[15] H.-N. Xuan, D. Zhang, and C. Wang, "Families of nontravelling wave solutions to a generalized variable coefficient two-dimensional KdV equation using symbolic computation," Chaos, Solitons and Fractals, vol. 23, no. 1, pp. 171-174, 2005.

[16] S. A. Elwakil, S. K. El-labany, M. A. Zahran, and R. Sabry, "New exact solutions for a generalized variable coefficients $2 \mathrm{D} \mathrm{KdV}$ equation," Chaos, Solitons and Fractals, vol. 19, no. 5, pp. 10831086, 2004.

[17] M. H. M. Moussa and R. M. El-Shiekh, "Direct reduction and exact solutions for generalized variable coefficients $2 \mathrm{D} \mathrm{KdV}$ equation under some integrability conditions," Communications in Theoretical Physics, vol. 55, no. 4, pp. 551-554, 2011.

[18] Y. Zhang, W.-W. Wei, T.-F. Cheng, and Y. Song, "Binary Bell polynomial application in generalized $(2+1)$-dimensional $\mathrm{KdV}$ equation with variable coefficients," Chinese Physics B, vol. 20, no. 11, Article ID 110204, 2011.

[19] N. H. Ibragimov, "A new conservation theorem," Journal of Mathematical Analysis and Applications, vol. 333, no. 1, pp. 311$328,2007$.

[20] N. H. Ibragimov, "Integrating factors, adjoint equations and Lagrangians," Journal of Mathematical Analysis and Applications, vol. 318, no. 2, pp. 742-757, 2006.

[21] N. H. Ibragimov, M. Torrisi, and R. Tracinà, "Self-adjointness and conservation laws of a generalized Burgers equation," Journal of Physics A, vol. 44, no. 14, Article ID 145201, 2011.

[22] N. H. Ibragimov, "Quasi-self-adjoint differential equations," Archives of ALGA, vol. 4, pp. 55-60, 2007.

[23] N. H. Ibragimov, "Nonlinear self-adjointness and conservation laws," Journal of Physics A, vol. 44, no. 43, Article ID 432002, 2011.

[24] N. H. Ibragimov, "Nonlinear self-adjointness in constructing conservation laws," Archives of ALGA, vol. 7-8, pp. 1-90, 2011. 
[25] C.-L. Bai, C.-J. Bai, and H. Zhao, "A new generalized algebraic method and its application in nonlinear evolution equations with variable coefficients," Zeitschrift fur Naturforschung A, vol. 60, no. 4, pp. 211-220, 2005.

[26] L.-H. Zhang, "Travelling wave solutions for the generalized Zakharov-Kuznetsov equation with higher-order nonlinear terms," Applied Mathematics and Computation, vol. 208, no. 1, pp. 144-155, 2009.

[27] X. Zhao, D. Tang, and L. Wang, "New soliton-like solutions for $\mathrm{KdV}$ equation with variable coefficient," Physics Letters $A$, vol. 346, no. 4, pp. 288-291, 2005.

[28] K.-J. Cai, C. Zhang, T. Xu, H. Zhang, and B. Tian, "Modified Exp-function method and variable-coefficient Korteweg-de Vries model from Bose-Einstein condensates," International Journal of Modern Physics B, vol. 24, no. 19, pp. 3759-3768, 2010.

[29] J. Li, B. Tian, X.-H. Meng, T. Xu, C.-Y. Zhang, and Y.-X. Zhang, "Variable-cofficient miura transforamation and integrable properties for a generalized variable-coefficeint korteweg-de vries equatiojn from boseeinstein condenstates with symbolic computiation," International Journal of Modern Physics B, vol. 23, no. 4, pp. 571-584, 2009.

[30] C. Huai-Tang and Z. Hong-Qing, "New double periodic and multiple soliton solutions of the generalized $(2+1)$-dimensional Boussinesq equation," Chaos, Solitons and Fractals, vol. 20, no. 4, pp. 765-769, 2004. 


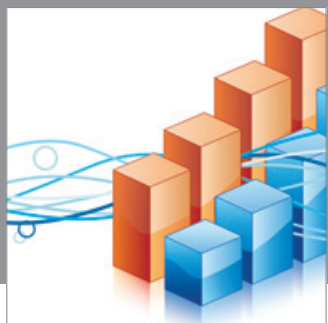

Advances in

Operations Research

mansans

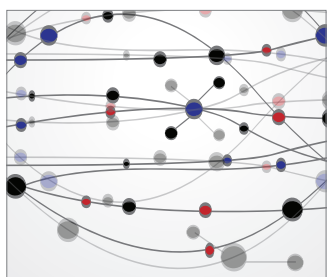

The Scientific World Journal
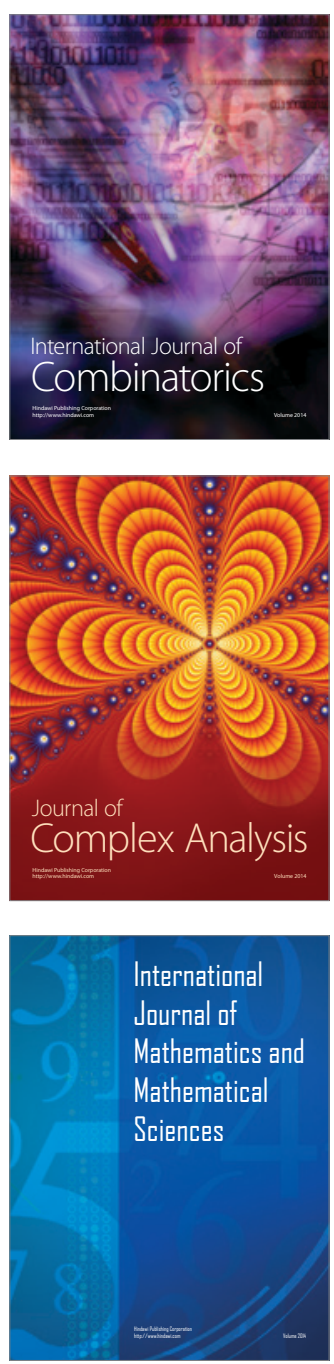
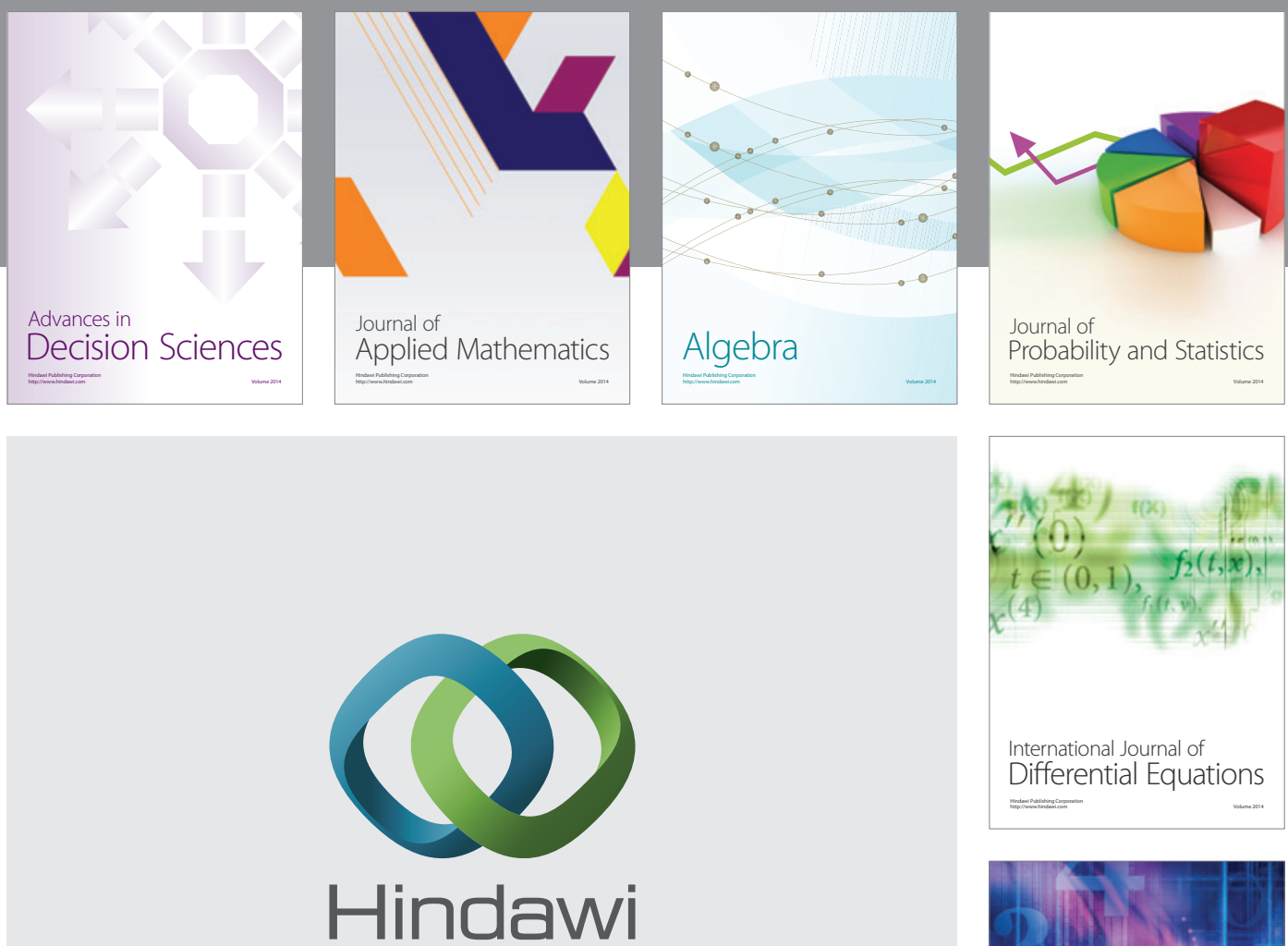

Submit your manuscripts at http://www.hindawi.com
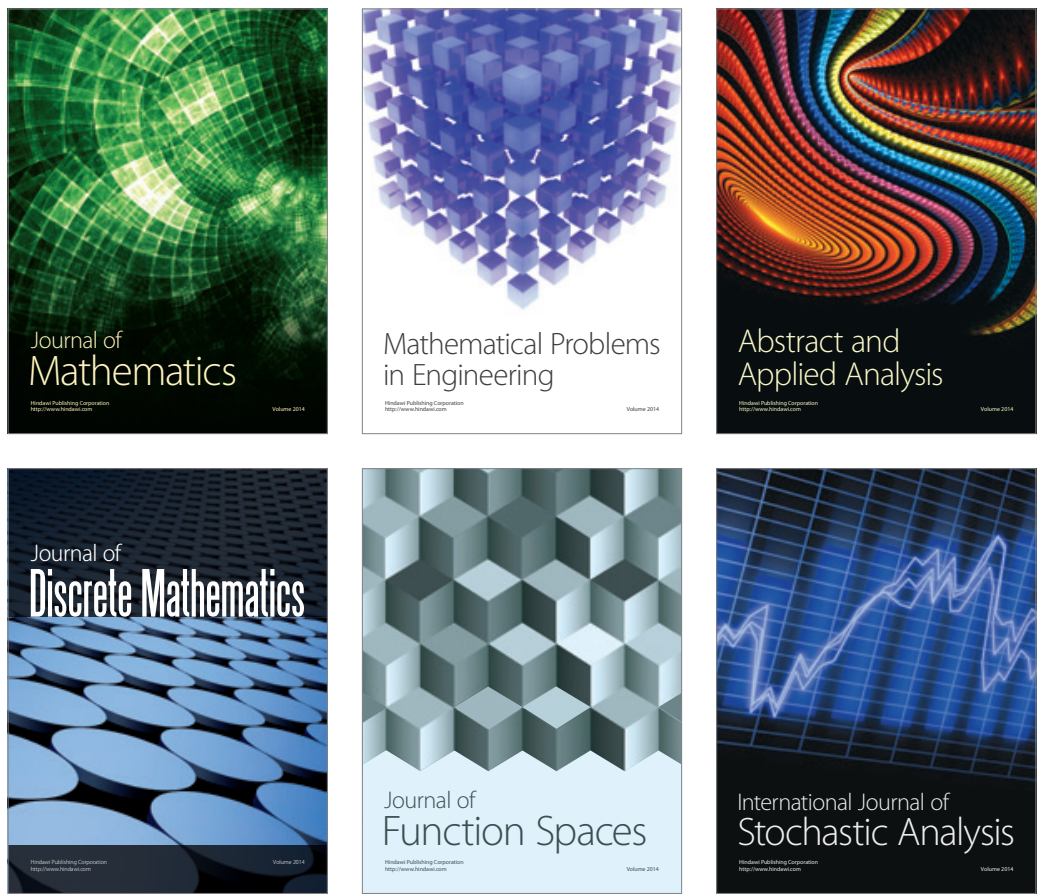

Journal of

Function Spaces

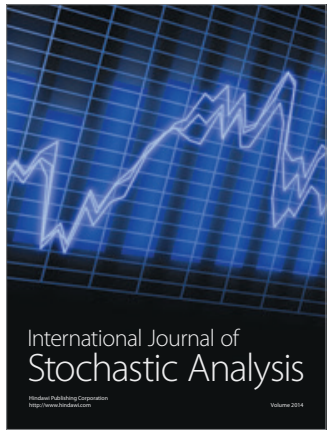

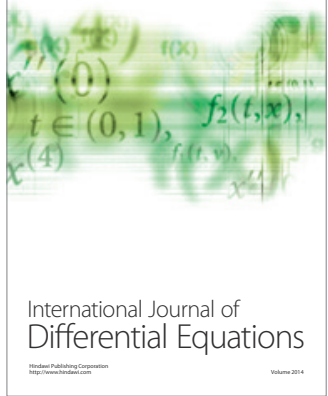
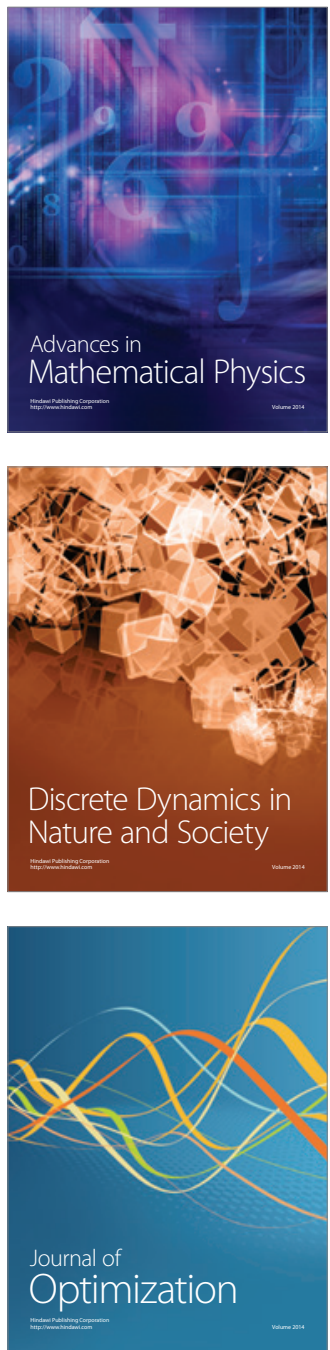